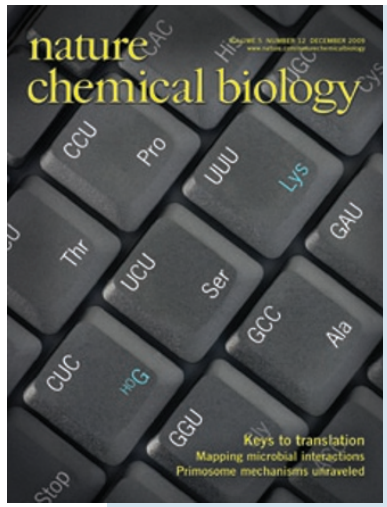

\section{Testing translation}

As reflected by the recent Nobel Prize in Chemistry, the intricacy and complexity of the ribosome continues to serve as an intellectual challenge and source of engineering inspiration. A critical step in protein translation is recognition of the acylated tRNA substrate. Though originally thought to rely purely on the interaction between the codon and anticodon, ongoing research has demonstrated that there are many subtleties involved. Effraim et al. investigated the role of the charged amino acid in the recognition process. Competition assays and single molecule studies revealed a small but significant difference in the ability of the ribosome to utilize correctly and incorrectly loaded residues. Kawakami et al. took advantage of these small distinctions by using a cell-free translation system in combination with a reactive three-amino-acid sequence to generate natural and nonnatural cyclic peptides and peptide libraries. These results should translate into new insights and methods for ribosomal research. [Brief Communication, p. 888; Article, p. 947]

\section{Vitamin-fortified RNA}

By virtue of their coding and structural properties, RNA molecules are central and active players in information flow and catalysis in cells. In some cases, post-transcriptional processing further tunes the properties of RNAs. For example, precise chemical modifications of tRNA or rRNA nucleotides are critical for the structure and function of these folded RNAs. Recent studies suggest that there may be more naturally occurring small molecule-RNA conjugates than previously thought. By subjecting cellular RNA to nuclease digestion and comparative LC/MS analysis, Chen et al. revealed that certain bacterial small RNAs (generally $<200$ nucleotides) are modified at their $5^{\prime}$ ends by the redox coenzyme NAD. The NAD moieties appear to be installed on RNA termini in a post-transcriptional process. Though the precise biosynthetic mechanisms leading to NAD-RNA conjugates await further analysis, the current study expands our view of modified cellular RNAs and provides impetus to explore their functional roles within modern biochemistry. [Brief Communication, p. 879]

\section{Resisting change}

Protein aggregates are hallmarks of several neurodegenerative diseases, and it is thought that different amyloid structures or 'strains' of the same protein can underlie distinct disease states. For example, the yeast prion protein Sup35 assembles differently based on temperature, though it is not clear which of these

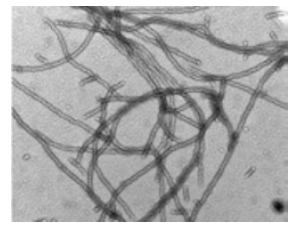
structures represents the prion $\left(\left[P S I^{+}\right]\right)$strain. Also, compounds that attenuate disease may need to affect more than one strain. The green tea catechin EGCG has distinct anti-amyloid activities from another compound, DAPH-12. Roberts et al. found that the spectrum of Sup35 infectious structures show differing sensitivities to EGCG. In vitro, EGCG blocked Sup35 prionogenesis, eliminated specific preformed Sup35

Written by Mirella Bucci, Catherine Goodman, Joanne Kotz \& Terry L. Sheppard

\section{IN THIS ISS UE}

prions and disrupted prion contacts; in vivo, it could attenuate weak $\left[P S I^{+}\right]$. EGCG also revealed a resistant strain that leads to strong $\left[P S I^{+}\right]$. Because of the strain-selective effects, synergistic combinations of EGCG and DAPH-12 were needed to alleviate multiple $\left[\mathrm{PSI}^{+}\right]$strains.

[Article, p. 936]

\section{Curtailing curli}

Biofilms are aggregates of microorganisms that are resistant to antibiotics and implicated in serious and persistent infections such as urinary tract infections (UTIs). Similarly to other enteric bacteria, biofilm formation in uropathogenic Escherichia coli (UPEC) is mediated by extracellular appendages including hair-like pili

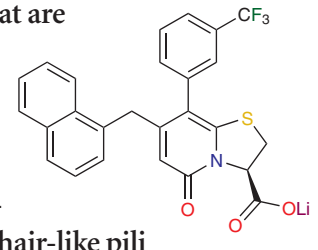
and amyloid fibers known as curli. Waksman, Hultgren, Almqvist and colleagues had previously identified a ring-fused 2-pyridone that inhibited pili biosynthesis. Starting from this pilicide compound, the authors identified analogs that inhibited curli assembly in a cellular assay while retaining pilicide activity. These curlicide compounds inhibited curli-dependent biofilm formation on plastic and at air-liquid interfaces. Using UPEC deletion strains, the authors first demonstrated the involvement of curli in bacterial colonization in a murine UTI model and then went on to show that the most potent curlicide significantly attenuated virulence in this in vivo model. [Article, p. 913]

\section{Gly-mpse into metalloenzyme design}

De novo design of enzymes remains a significant challenge. Lombardi, DeGrado and colleagues have previously designed 'Due Ferri' proteins that are dimers composed of helixloop-helix monomers. This family of proteins binds two $\mathrm{Fe}$ (II) ions that can react rapidly

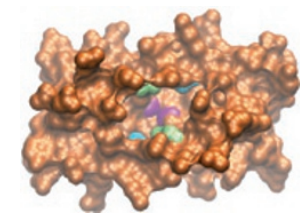
with $\mathrm{O}_{2}$ to form an oxo-bridged di-Fe(III) species. Faiella et al. mutated two leucine residues in the first helix to glycines, which introduced a phenol binding pocket near the di-iron site but significantly destabilized the protein. Through statistical analysis of the Protein Data Bank, the loop sequence in the protein was optimized to compensate for this loss of stability. An NMR structure of the resulting protein with di- $\mathrm{Zn}$ (II) bound revealed that the structure was nearly identical to the model. The designed enzyme catalyzed the oxidization of a range of phenols, thus demonstrating that the metalloprotein was successfully converted to a metalloenzyme. [Brief Communication, p. 882]

\section{Predator and prey on the move}

In microbial ecosystems, cell-to-cell communication occurs through direct cell-cell contact or through small diffusible chemicals. Chemicalmediated interactions can contribute to biodiversity both mutualistically and competitively and can occur over large spatial distances. Reducing cellular motility also promotes biodiversity, but the connection between the more long-range chemical signals and shorter-range motile events in modulating biodiversity is unclear. To test the connection, Song et al. used an engineered Escherichia coli predator-prey ecosystem mediated by quorum sensing that resembles many natural chemical-mediated competitive ecosystems. They found that motility had differing effects on biodiversity based on the spatiotemporal locations of the predator and the prey. Mathematical modeling revealed a complex interplay between cellular motility, chemical diffusion and habitat configuration in the chemical-mediated system. [Article, p. 929] 